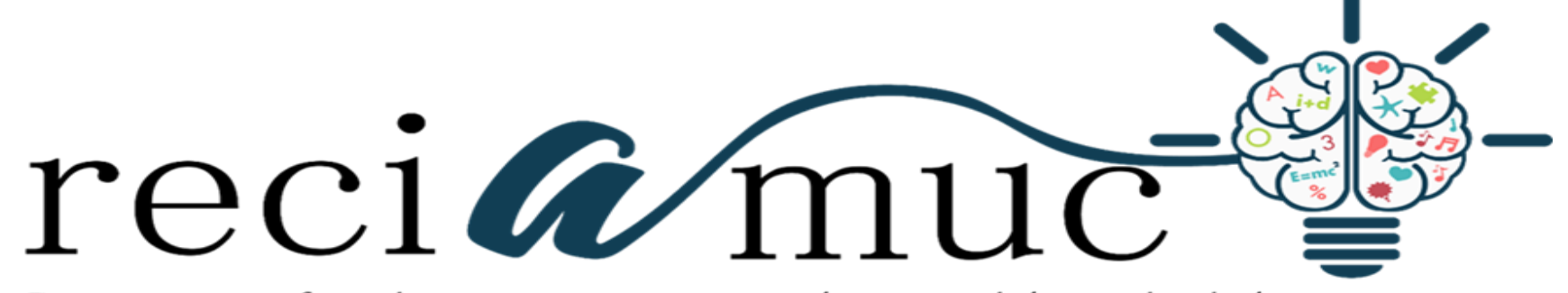

Revista científica de investigación actualización del mundo de las ciencias

Francisco Xavier Hugo Cárdenas a ; Carlos Roberto Flores Ramos '; Álvaro Raúl Peralta Beltrán ${ }^{c}$; Paul Eduardo Lara Pazos ${ }^{\mathrm{d}}$

Sostenibilidad empresarial en relación a los objetivos del desarrollo sostenible en el Ecuador

Corporate sustainability in relation to the objectives of sustainable development in Ecuador

Revista Científica de Investigación actualización del mundo de las Ciencias. Vol. 3 núm., 1, enero, ISSN: 2588-0748, 2018, pp. 670-699

DOI: $10.26820 /$ reciamuc/3.(1).enero.2019.670-699

URL: http://reciamuc.com/index.php/RECIAMUC/article/view/253

Código UNESCO: 5307.04 Estudios del desarrollo Económico

Tipo de Investigación: Artículo de Revisión

Editorial Saberes del Conocimiento

Recibido: 10/12/2018

Aceptado: 20/01/2019

Publicado: 30/01/2019

Correspondencia: director@ reciamuc.com

a. Magister Seguridad Salud y Ambiente; Ingeniero Ambiental; Fcohugo@ hotmail.com

b. Magister Seguridad Salud y Ambiente; Ingeniero Industrial; gerenciatecnica@gestionintegral.com

c. Magister Seguridad Salud y Ambiente; Doctor en Psicología Industrial; alvaroperalt@ gmail.com

d. Magister en Gestión en la Industria de los Hidrocarburos; Ingeniero de Petróleos; edu65lara@ yahoo.es 


\section{Sostenibilidad empresarial en relación a los objetivos del desarrollo sostenible \\ en el Ecuador}

Vol. 3, núm. 1., (2019)

Francisco Xavier Hugo Cárdenas; Carlos Roberto Flores Ramos; Álvaro Raúl Peralta Beltrán;

Paul Eduardo Lara Pazos

\section{RESUMEN}

Las naciones para lograr cambios progresivos en sus estructuras económicas, políticas, educativas, sociales y culturales, necesitan de grupos humanos encargados de trabajar conjuntamente con los planes propuestos por el Estado, esto permite, gestionar los respectivos cambios que la población en general necesita para incrementar sus capacidades productivas y mantener niveles de calidad competitivos en todos los sectores que aseguren su propia sostenibilidad. Por ello, las empresas se convierten en espacios fundamentales para alcanzar los objetivos o planes gubernamentales, encargados de fomentar progresivamente una relación flexible con las actuales tendencias y de este modo, convertirlas en instituciones que enfrenten los desafíos relacionados con la sostenibilidad de sus cadenas de valor, es decir, ciclo de vida completo de un producto o servicio. Estas ideas, llevan a entender que la intervención empresarial desde un contexto sostenible, asegura con el cumplimiento de las regulaciones, en materia de recursos para sus operaciones diarias o la gestión de necesidades y expectativas de sus grupos de interés, esto permite destacar una perfecta relación con los objetivos propuestos en los proyectos de desarrollo sostenible nacionales. En otras palabras, son desafíos que impactan a todo gobierno, empresa, organizaciones no gubernamentales y consumidores por igual, en consecuencia, hay que trabajar simultáneamente para evaluar de manera que se resuelvan los problemas ambientales y sociales a fin de transformar los productos o servicios y hacer la mejorar de la sostenibilidad del país. Según las consideraciones anteriores, surge la realización de este artículo, cuyo objetivo es analizar la sostenibilidad empresarial en relación a los objetivos del desarrollo sostenible en el Ecuador, para así ubicarlo en el marco de una investigación documental monográfica, apoyada en fuentes documentales que hacen posible la recopilación de información relevantes para finalmente elaborar las apreciaciones generales en cuanto al discurso realizado en su contenido teórico.

Palabras Claves: Sostenibilidad Empresarial; Objetivos; Desarrollo Sostenible Ecuador. 


\section{Sostenibilidad empresarial en relación a los objetivos del desarrollo sostenible en el Ecuador}

Vol. 3, núm. 1., (2019)

Francisco Xavier Hugo Cárdenas; Carlos Roberto Flores Ramos; Álvaro Raúl Peralta Beltrán; Paul Eduardo Lara Pazos

\section{ABSTRACT}

Nations to make progressive changes in their economic, political, educational, social and cultural structures, need for human groups to work together with the plans proposed by the State, this allows to manage the respective changes that people in general need to increase its production capacities and maintain competitive levels of quality in all sectors that will ensure their own sustainability. Therefore, companies become fundamental areas to achieve the goals or governmental plans, progressively encourage a flexible relationship with current trends and thus turn them into institutions that face the challenges related to the sustainability of their value chains, i.e., full life cycle of a product or service. These ideas lead to understand that corporate intervention from a sustainable context, ensures compliance of the regulations, in resources for their daily operations or management of needs and expectations of its stakeholders, this it allows to highlight a perfect relationship with the objectives proposed in the national sustainable development projects. In other words, they are challenges that impact all Government, business, NGOs and consumers alike, therefore we must work simultaneously to evaluate so that the environmental and social problems to be solved in order transform the products or services and make improve the sustainability of the country. In other words, they are challenges that impact all Government, business, NGOs and consumers alike, therefore we must work simultaneously to evaluate so that the environmental and social problems to be solved in order transform the products or services and make improve the sustainability of the country. According to the above considerations, arises the realization of this article, whose objective is to analyze the corporate sustainability in relation to the objectives of sustainable development in the Ecuador, to so place him in the context of a documentary research monographic, supported in documentary sources that make possible the collection of relevant information to finally make general appreciations as for the speech made in its theoretical contents.

Key Words: Business Sustainability; Goals; Sustainable Development Ecuador. 


\section{Sostenibilidad empresarial en relación a los objetivos del desarrollo sostenible \\ en el Ecuador}

Vol. 3, núm. 1., (2019)

Francisco Xavier Hugo Cárdenas; Carlos Roberto Flores Ramos; Álvaro Raúl Peralta Beltrán;

Paul Eduardo Lara Pazos

\section{Introducción.}

En las empresas, los procesos administrativos hacen posible preparar el terreno para garantizar las condiciones en virtud de ofrecer calidad durante la prestación de un servicio o venta de diversos productos que ayudan a lograr cambios progresivos en la vida colectiva dentro de una sociedad cualquiera. Es allí, donde el administrador como gerente encargado de hacer cumplir dichas tareas, asume con responsabilidad el adecuar el entorno donde se circunscribe la organización conjuntamente con su talento humano, a fin de mantener niveles armónicos que le ayuden a realizar las actividades de manera flexible y garantizar éxito organizacional.

En consecuencia, las empresa en el siglo XXI, a nivel mundial, se enfrentan, con escenarios altamente complejos en cuanto a retos precisos en el área del conocimiento, así como en el aprendizaje continuo que les exige modelar conductas, que permitan garantizar un ambiente confortable que promueva la transformación empresarial, de allí, que actualmente para mantener su competitividad en el entorno inmediato, deben desarrollar criterios de servicio encargados de ofrecerles condiciones viables para adoptar el cambio de sus objetivos, metas y visión, esto hace posible corresponder a la visión económica que el país visualice como parte esencial del crecimiento externo e interno.

De este modo, se precisa que las empresas para cumplir con su razón social, deben mirar detenidamente el complejo mundo externo donde se encuentra insertada, para ello, es necesario recurrir a un manejo efectivo del recurso humano, esta acción le permite garantizar de forma significativa su sostenibilidad, en otras palabras, las exigencias de los cambios que suelen ofrecer oportunidades continuas, hacen posible acelerar la estructura empresarial con el fin de 


\section{Sostenibilidad empresarial en relación a los objetivos del desarrollo sostenible en el Ecuador}

Vol. 3, núm. 1., (2019)

Francisco Xavier Hugo Cárdenas; Carlos Roberto Flores Ramos; Álvaro Raúl Peralta Beltrán; Paul Eduardo Lara Pazos

convertirlas en eficaces y eficientes para enfrentar cualquier reto que el fenómeno de globalización les presente.

De lo antes expuesto, es importante citar lo planteado por Mariño (2018) "la sostenibilidad empresarial busca un equilibrio entre lo económico, social y ambiental". (p.25). La definición, asegura que mediante este concepto se puede concebir la actividad empresarial, como una visión donde el recurso humano, no puede solo gestionar los recursos financieros y materiales; sino mirar el entorno inmediato con el fin de asegurar el respectivo equilibrio de la organización con el medio, a fin de ofrecer a la población servicios de calidad, sin generar cambios en el ambiente, todo lo contrario proporciona competitividad desde un contexto ambiental armónico.

Según lo planteado, se entiende que mediante la sostenibilidad empresarial cada organización logra mantener un estilo de vida muy corporativo cuya meta es el balance entre la creación, riqueza y el uso de diferente recurso humano, material, natural y económico. Es decir, De allí, que el objetivo final de la empresa no es más que mejorar las condiciones socioeconómicas para todos, lo que incluye, por supuesto, a las empresas mismas. Por eso una empresa es sustentable cuando tiene la capacidad de asegurar su continuidad, posicionamiento a largo plazo, además contribuir al progreso de la generación presente y futura. Aunado a esto, Mariño (ob.cit), destaca que "quedó atrás esa visión en donde el único propósito era generar la mayor riqueza posible para los dueños del capital”. (p.28). 


\section{Sostenibilidad empresarial en relación a los objetivos del desarrollo sostenible \\ en el Ecuador}

Vol. 3, núm. 1., (2019)

Francisco Xavier Hugo Cárdenas; Carlos Roberto Flores Ramos; Álvaro Raúl Peralta Beltrán;

Paul Eduardo Lara Pazos

Lo citado, permite interpretar el cambio significativo que las empresas modernas tienen hoy, concebir sus funciones desde una perspectiva transformadora, concebir el trabajo como el mecanismo idóneo para generar la producción, proporcionar un servicio ajustado a las necesidades presente en el entorno, de este modo, no sólo reconoce los derechos laborales, humanos que atentan contra el medio ambiente, sino, hay una convicción social encargada de visualizar temar o entidades que velen por el cumplimiento de las normas. Por lo tanto, es importante analizar las variables que son clave para la competitividad de las empresas, a fin de lograr la respectiva sostenibilidad como fuente de ventajas competitivas.

De estas ideas, se precisa que al hablar de sostenibilidad empresarial hoy en día, es entender el compromiso de las empresas con su stakeholders, y de la mirada hacia el futuro de las organizaciones, no sólo en términos de viabilidad o éxito financiero, sino también en clave social y medioambiental. Una empresa sostenible añade valor internamente y en su entorno, es aquella que genera un valor compartido con todos sus stakeholders. Para ello, se hace necesario que la sostenibilidad se integre en todos los procesos de toma de decisiones, planificación, gobierno y rendición de cuentas, mediante la identificación de quiénes son los stakeholders prioritarios y cómo dar respuesta a sus expectativas, articulando canales de comunicación efectivos que favorezcan el diálogo y transparencia, incorporando las cuestiones materiales en el modelo de gestión y desarrollar estrategias que estén alineadas con la sostenibilidad.

En consecuencia, la sostenibilidad empresarial, va más allá de llevar un manejo eficiente del flujo de caja e invertir en diversos campos. Es una definida política de negocio en la que 


\section{Sostenibilidad empresarial en relación a los objetivos del desarrollo sostenible en el Ecuador}

Vol. 3, núm. 1., (2019)

Francisco Xavier Hugo Cárdenas; Carlos Roberto Flores Ramos; Álvaro Raúl Peralta Beltrán; Paul Eduardo Lara Pazos

debe haber unidad, coherencia, continuidad y viabilidad. Esto, permiten valorar el significado que posee, el entorno inmediato para una empresa, al momento de considerar sus objetivos como parte esencial del cambio, en otras palabras, simplemente corresponder su visión y misión con las necesidades humanas, materiales y económicas que cada nación tiene de sus diferentes instituciones públicas y privadas, para así fijar posiciones competentes ante posibles transformaciones políticas, sociales, económicas, financieras, educativas y culturales.

De lo antes expuesto, se entiende que existe una vinculación directa entre la sostenibilidad de las empresas con el desarrollo sostenible de una sociedad, pues, las primeras ofrecen oportunidades comunes en pro de alcanzar objetivos afines, y el segundo fija posiciones claras en cuanto a las necesidades que la población en general posee y por lo tanto, debe existir una compaginación entre los sectores productivos a fin de canalizar los problemas e introducir los respectivos cambios que le ofrecerán a la nación un recorrido por nuevas vías para ofrecer mejores expectativas a los ciudadanos. Según lo citado, Ruiz (2017) destaca que "cada nación construye su desarrollo sostenible en función a la realidad de sostenibilidad que poseen sus empresas, hay que elevar el trabajo colectivo como indicador de cambios puntuales en las esferas sociales". (p.22)

Dentro de este marco de ideas, los países Latinoamericanos como resultado del crecimiento población, cambios en las visiones políticas, la búsqueda de implementar nuevas estrategias encargadas de facilitar el desarrollo sostenible, crean condiciones viables para reconocer en sus empresas posibles herramientas esenciales que le abren el camino para asegurar su respectivo crecimiento económico, social, político, educativo entre otros. Por ello, gestionan 


\section{Sostenibilidad empresarial en relación a los objetivos del desarrollo sostenible \\ en el Ecuador}

Vol. 3, núm. 1., (2019)

Francisco Xavier Hugo Cárdenas; Carlos Roberto Flores Ramos; Álvaro Raúl Peralta Beltrán;

Paul Eduardo Lara Pazos

mediante medidas gubernamentales el cumplimiento de los objetivos del desarrollo sostenible; ejemplo de ello Ecuador, que se incursa en la introducción de un nuevo paradigma político, dirigido a lograr el crecimiento no sólo de las empresas públicas; sino, que conduce a propiciar condiciones generadoras de medidas proclives a lograr la inserción de las empresas privadas como pilares esenciales para hablar de desarrollo sostenible.

En este particular, el Programa de las Naciones Unidas para el Desarrollo Ecuador (2019) tiene como visión:

Impulsar las capacidades y esfuerzos nacionales para construir una sociedad equitativa a través de la reducción de la pobreza, promoción de los derechos humanos y la gobernabilidad democrática. Vincular al país con conocimientos, experiencias y recursos en alianza con los asociados que permitan promover la ampliación de las oportunidades a las ecuatorianas y ecuatorianos. (p.6)

Lo anteriormente expuesto, permite precisar que Ecuador como nación al estar incursa en el programa de las naciones unidas para el desarrollo, mantiene en sus políticas externas e internas una visión centrada en lograr la transformación general del país, para lo cual, asume las experiencias mediante alianzas abiertas que le aseguren promover la ampliación de oportunidades para la población, de este modo, requiere que las empresas, comprendan el significado de adecuar sus funciones bajo una visión de sostenibilidad a fin de crear las condiciones óptimas y competitivas que facilitarán el ingreso de recursos financieros, productos e innovación en los diferentes sectores que estructuran al país. 


\section{Sostenibilidad empresarial en relación a los objetivos del desarrollo sostenible en el Ecuador}

Vol. 3, núm. 1., (2019)

Francisco Xavier Hugo Cárdenas; Carlos Roberto Flores Ramos; Álvaro Raúl Peralta Beltrán; Paul Eduardo Lara Pazos

Asimismo, Correa (2015), indica que necesario "romper las inercias conceptuales, transitar hacia nuevas métricas que desafíen el paradigma tradicional, se ha de medir el trabajo no remunerado, y no solo el del mercado, calidad de vida general y producto interno bruto per cápita". (p.6). Lo planteado, lleva a comprender que la política de Ecuador, es mirar las situaciones que viven la población, buscar mediante el apoyo de las empresas un desarrollo sostenible que asegure su crecimiento como país, por ello, es relevante no sólo concebir las estadísticas en cuestiones del ingreso per cápita, sino valorar las condiciones reales que tienen los trabajadores en las diferentes actividades productivas, comerciales y laborales con el fin de afianzar cambios progresivos en su calidad de vida.

No obstante a pesar de las diferentes propuestas realizadas por el gobierno ecuatoriano, se miran situaciones contradictorias con respecto a la sostenibilidad empresarial, es así como Acción Ecológica Ecuador (2015) respalda el hecho de las empresas "ocasionan daños al ambiente y al entorno social, crean efectos a los pueblos indígenas y campesinos en las provincias de Esmeralda y Pichincha”. (p.10). Estos datos, permiten replantearse la necesidad de gestionar cambios específicos en los programas de gobiernos, a fin de profundizar en las consecuencias que tiene la falta de una empresa con sentido de sostenibilidad, es decir, es importante concebir el trabajo empresarial, desde la visión de crear condiciones ambientales que promocionen niveles de vida ajustado a una calidad, para así ser cada vez más competitivo en las actividades promocionadas durante la realización de un trabajo. Pues, al existir correspondencia, en estos criterios se aumentan las posibilidades de ofrecer a los ecuatorianos y ecuatorianas 


\section{Sostenibilidad empresarial en relación a los objetivos del desarrollo sostenible \\ en el Ecuador}

Vol. 3, núm. 1., (2019)

Francisco Xavier Hugo Cárdenas; Carlos Roberto Flores Ramos; Álvaro Raúl Peralta Beltrán;

Paul Eduardo Lara Pazos

cambios progresivos en sus actividades educativas, laborales, sociales, salud, culturales entre otras.

Estas ideas, sirven de referencia para el desarrollo de este artículo, mediante el cual se busca analizar aspectos relativos a la sostenibilidad empresarial en relación a los objetivos del desarrollo sostenible en el Ecuador, por ello, es importante encaminar el proceso hacia la identificación del método científico como fuente esencial encargada de dar las respectivas acciones que permiten la construcción del conocimiento desde una visión innovadora y al mismo tiempo, propicia una selección cónsona de las técnicas documentales para establecer los mecanismos básicos que dan cabida al desarrollo del tema. Dado que, una empresa sostenible proporciona a la sociedad en general valor económico, medioambiental y social a corto y largo plazo, acción que repercute en la ciudadanía.

\section{Método.}

En el campo científico las acciones concernientes para la revisión de los contenidos, están directamente vinculados con la incorporación de un método que asegure el desarrollo de los propósitos que caracterizan al tema seleccionado, por ello, el presente artículo se apoya en lo citado por, Naranjo (2019) “un método ayuda a elaborar consideraciones generales que permiten indicar en forma clara nuevas ideas, las cuales complementan el trabajo científico". (p.63)

De acuerdo con lo citado, por el autor se puede indicar que para dar la respectiva acción científica al artículo se procede a seleccionar el método de diferencias que según Naranjo (ob.cit) su procedimiento consiste en "reunir varios casos en los que se observen eventos que producen 


\section{Sostenibilidad empresarial en relación a los objetivos del desarrollo sostenible en el Ecuador}

Vol. 3, núm. 1., (2019)

Francisco Xavier Hugo Cárdenas; Carlos Roberto Flores Ramos; Álvaro Raúl Peralta Beltrán; Paul Eduardo Lara Pazos

un efecto para luego explicarlo en forma general". (p.43). Lo citado, lleva a entender que mediante dicha aplicación del método diferencias, se puede fijar posiciones claras ante los distintos aspectos que están directamente relacionados con la sostenibilidad empresarial en relación con el desarrollo sostenible en el Ecuador

Tipo de Investigación

Al buscar desarrollar los eventos que caracterizan el marco metodológico en una investigación, se hace necesario partir de la identificación del tipo, en cuanto al estudio que se lleva a cabo, se encuentra determinado por la definición dada por Mendoza (2017), la investigación documental "desarrolla actividades concernientes a la interpretación de problemas planteados a nivel teórico, la información requerida para abordarlos se encuentra básicamente en materiales impresos, audiovisuales y /o electrónicos" (p.41).

En razón de esta definición, se puede indicar que al fin de cumplir con las características previas que identifican al trabajo, se realizaron una serie de actividades concernientes a la revisión de fuentes primarias referidas a los aspectos fisiopatológicos en pacientes con problemas de tiroides, para luego ajustar su selección correspondiente a las necesidades de investigación. Para ello, implementó las condiciones que determinan su nivel descriptivo, que según Mendoza (ob.cit), "es apreciar, desglosar e indicar en forma analítica todos los hechos de un problema" (p.55).

Técnicas Documentales 


\section{Sostenibilidad empresarial en relación a los objetivos del desarrollo sostenible \\ en el Ecuador}

Vol. 3, núm. 1., (2019)

Francisco Xavier Hugo Cárdenas; Carlos Roberto Flores Ramos; Álvaro Raúl Peralta Beltrán; Paul Eduardo Lara Pazos

Con el fin de ajustar las diferentes apreciaciones dentro de un orden, coherencia y pertinente, es importante buscar mecanismos idóneos que aseguren la continuidad del proceso investigativo, por ello, es relevante incluir las técnicas documentales como apoyo bibliográfico encargado de dar la respectiva flexibilidad al contenido. Mendoza (ob.cit), consiste "en interpretar en forma analítica el contenido de un texto para exponer ideas coherentes y vinculantes entre sí” (p.54).

De igual manera, se puede indicar que mediante la utilización de las técnicas documentales sirvió para la confrontación de las ideas, que hicieron posible tomar aquellos aspectos generales para separarlos en parte y conformar sus propias apreciaciones individuales. Es decir, se desarrollaron los contenidos en forma generales para luego conformar una estructura global de los hechos a tratar.

\section{Técnicas de Recolección de Información}

La continuidad de las actividades metodológicas llevan a la realización de un conjunto de tareas previas para indicar finalmente ubicar el contenido dentro de una acción interpretativa, es por ello, que se seleccionan técnicas basadas en la lectura como elemento esencial para dar la respectiva organización de las informaciones desde una perspectiva general para llegar a lo particular y retroceder en el otro sentido para conocer las realidades en su totalidad. De allí, que se procedió a identificar los elementos generales que caracterizan el tema una vez interpretado fueron compaginados en forma adecuada hasta responder a cada de las situaciones encontradas en su desarrollo. 


\section{Sostenibilidad empresarial en relación a los objetivos del desarrollo sostenible en el Ecuador}

Vol. 3, núm. 1., (2019)

Francisco Xavier Hugo Cárdenas; Carlos Roberto Flores Ramos; Álvaro Raúl Peralta Beltrán; Paul Eduardo Lara Pazos

Sin embargo, el trabajo del método al ser acompañado por una técnica, como la lectura rápida, crítica y reflexiva como alternativas para la revisión y selección final de los aspectos de interés, a criterio de Mendoza (ob.cit) "son acciones combinadas para ayudar al investigador a detectar los materiales y adecuarlos a sus propios intereses" (p.78). Una vez cumplida las etapas anteriores se realizaron un conjunto fases todas interconectadas para responder efectivamente a los planteamientos generales.

Por lo tanto, se estimaron las condiciones generales que guardan relación con el tema en estudio, para luego apreciar desde la revisión de aquellos recursos materiales, libros, revistas, periódicos y recursos tecnológicos los eventos contentivos a los aspectos a investigar. Para así, llevar a construir en forma clara los aportes generales que caracterizan una situación o contenido viable para ser estudiado y proporcionar una visión de las apreciaciones particulares que dan cabida a interpretaciones generales para así llegar a construir las conclusiones.

En consecuencia, para ampliar los aspectos generales que fueron estimados con anticipación como interés del presente artículo, se llega al desarrollo de los resultados encargados de proporcionar las respectivas orientaciones que caracterizan a los aspectos fisiopatológico en pacientes con problemas de tiroides. Es decir, una vez cumplida la revisión, selección y preparación del material a insertar en el contenido del artículo investigación, se procedió a la elaboración y desarrollo de los diferentes elementos funcionales que caracterizan al tema con ello, se pudo darle al estudio el respectivo cuerpo interpretativo necesario para su valoración científica.

\section{Resultados.}




\section{Sostenibilidad empresarial en relación a los objetivos del desarrollo sostenible \\ en el Ecuador}

Vol. 3, núm. 1., (2019)

Francisco Xavier Hugo Cárdenas; Carlos Roberto Flores Ramos; Álvaro Raúl Peralta Beltrán; Paul Eduardo Lara Pazos

Cada uno de los aspectos que se desarrollan a continuación, guardan relación con los contenidos teóricos que sirven de referencia documental al artículo, los mismos son presentados en función a la vinculación entre las características que identifican al fenómeno en estudio.

\section{Sostenibilidad Empresarial}

Al hacer referencia a la sostenibilidad empresarial es más que un concepto que ha tomado fuerza en los últimos años, es un estilo de gobierno organizacional que fomenta el equilibrio entre lo económico, social y ambiental. Por lo tanto, se encuentra representada por aquellas que ofertan bienes o servicios que generan impactos ambientales positivos, incorporando buenas prácticas económicas, sociales y ambientales con enfoque de ciclo de vida, contribuyendo a la conservación del ambiente como capital natural que soporta el desarrollo del territorio. También se consideran sostenibles las empresas o ideas que, aunque su razón de ser no esté relacionada con el cuidado del medio ambiente y su relación con el entorno, realizan acciones que benefician al medio ambiente y comunidades circundantes a su quehacer.

Es importante acotar que, esto no consiste en hacer acciones enmarcadas en bondades, simplemente se trata de realizar actuaciones empresariales que ayuden a la preservación del medio ambiente y mejoren el estilo de vida de las comunidades que rodean la empresa, por lo que hay una oportunidad de ayudar y de generar más riquezas. Cabe agregar, que algunas de las grandes empresas han implementado tecnología que conllevan al uso de luz natural, ahorro del 


\section{Sostenibilidad empresarial en relación a los objetivos del desarrollo sostenible en el Ecuador}

Vol. 3, núm. 1., (2019)

Francisco Xavier Hugo Cárdenas; Carlos Roberto Flores Ramos; Álvaro Raúl Peralta Beltrán; Paul Eduardo Lara Pazos

agua, manejo de aire acondicionado y al uso de residuos. En el aspecto social diseñado programas de bienestar para las comunidades, talleres de empleabilidad, construcción de escuelas, entre otras. Esto sumado a que dentro de su estrategia y actividades de sostenibilidad empresarial, han entendido la importancia de esta cultura, por lo que implementa acciones como el teletrabajo, incentivan a los empleados a que usen bicicleta, carro compartido y transporte público para ir a trabajar.

Por otro lado, los consumidores se preocupan por comprar productos que aporten a la reducción de los niveles de contaminación, por lo que al adquirirlos sienten que están contribuyendo directamente con el ecosistema. Para que esto sea realmente efectivo, es importante diseñar programas de Responsabilidad Social Corporativa, pues, no solo aporta a esto, sino que mejora la imagen de la empresa, implica a los colaboradores en estas acciones, incorpora valor a la empresa y fideliza a los clientes al sentirse identificados con esta visión. De acuerdo, con las características e importancia del desarrollo sostenible para las empresas, Andrade (2018) precisa algunos beneficios que puedes obtener de implementar estas accione:

Genera buena reputación e imagen de marca al ayudar a los demás. Ahorra costos al tener un negocio sostenible optimiza los recursos. Recibe exenciones fiscales al realizar acciones de sostenibilidad, las empresas pueden ser exentas de impuestos relacionados con el manejo de residuos y demás. Facilita el acceso a nuevos mercados y entidades. Mejora el clima laboral, pues, los trabajadores suelen comprometerse y sentirse identificados con acciones socialmente solidarias. Hay oportunidad de ayudar al diseñar programas que apoyen a las comunidades más 


\section{Sostenibilidad empresarial en relación a los objetivos del desarrollo sostenible \\ en el Ecuador}

Vol. 3, núm. 1., (2019)

Francisco Xavier Hugo Cárdenas; Carlos Roberto Flores Ramos; Álvaro Raúl Peralta Beltrán;

Paul Eduardo Lara Pazos

vulnerables. Se producen ingresos que aprovechan otro tipo de recursos y materiales más económicos, adicionalmente, a que los consumidores compran más a este tipo de empresas. (p.32)

Cada una de las acciones citadas, llevan a comprender que la presencia de empresas sostenibles, garantiza en el medio donde se encuentran, cambios progresivos que benefician a la población en general, pues, se incrementa la posibilidad de ser más competitivas hacia los consumidores, dado que, generan confianza en ellas y busca mediante su uso en relación a los productos o servicios para así trasladar los beneficios a la ciudadanía de forma más coherente a sus necesidades.

De lo antes citado, se comprende que ser sostenible es desarrollar la empresa teniendo en cuenta los impactos medioambientales y sociales que generan cada acción que realiza, de allí la importancia de tener administradores conscientes de la manera en la que se desarrollan los productos y la forma como las comunidades lo perciben. En otras palabras, cada gerente y trabajador debe estar comprometido con el hacer, saber y emplear las estrategias que le darán mejores oportunidades a todos, en perfecta relación con el medio ambiente y sus comunidades.

Por otra parte, es importante visualizar que las empresas al buscar la sostenibilidad deben mirar sus acciones gerenciales en tres vértices: económico, ambiental y social. De su combinación, logra agregar estrategias trianguladas que dirigen las actividades empresariales hacia la realización de mantener un impacto reducido en el ambiente, empleados felices; además de añadir un valor a la empresa. En este sentido, Gómez (2018) describe las condiciones generales de estos aspectos: 


\section{Sostenibilidad empresarial en relación a los objetivos del desarrollo sostenible en el Ecuador}

Vol. 3, núm. 1., (2019)

Francisco Xavier Hugo Cárdenas; Carlos Roberto Flores Ramos; Álvaro Raúl Peralta Beltrán; Paul Eduardo Lara Pazos

Sostenibilidad Económica: Hace referencia al crecimiento económico que sostiene a la empresa, siempre con respecto a los recursos naturales, reducción progresiva de la huella ecológica de los productos en su ciclo completo y riqueza distribuida de forma equitativa. Es importante que el gerente evalúe: necesidades, deuda y estructura de endeudamiento, liquidez general, rentabilidad liquida, operacional de ventas, activos entre otros.

Sostenibilidad Ambiental: Busca minimizar los impactos ambientales negativos producto de la actividad empresarial con el objetivo de crear con el tiempo impactos positivos. Para ello, se base en una manera de abordar el ciclo de vida del producto, interiorizando las buenas prácticas a todos los niveles de la estructura interna de la empresa. Más que una estrategia de marketing, se debe ver como un incentivo para la innovación y capacidad de adaptación de la empresa. Debe buscar: calidad del producto, cantidad de energía usada, uso de equipamiento, materiales reutilizables.

Sostenibilidad Social: Plasma que la empresa debe buscar la dimensión humana, al visualizar las condiciones externas e internas de la empresa que influyen en lo que sucede en los empleados, valores, clientes y comunidades locales, en consecuencia, es importante gestionar estos impactos de manera proactiva, buscar que las personas sean felices y comunidades más desarrolladas. Por lo tanto, es considerable los siguientes indicadores: voluntariado, programas de apoyo a la comunidad, inversión e intervención en la comunidad, puestos de trabajos creados, diversidad de los empleados.

Cada uno de los aspectos estructurales de la sostenibilidad empresarial, lleva a precisar que mediante la identificación de estos indicadores, se lograr estimular cambios importantes en 


\section{Sostenibilidad empresarial en relación a los objetivos del desarrollo sostenible \\ en el Ecuador}

Vol. 3, núm. 1., (2019)

Francisco Xavier Hugo Cárdenas; Carlos Roberto Flores Ramos; Álvaro Raúl Peralta Beltrán; Paul Eduardo Lara Pazos

sus estructuras administrativas, además, los gerentes como actores sociales miran el entorno no como un espacio limitante, sino todo lo contrario, agilizan acciones colectivas que sean incorporadas de manera individual para así asegurar el desarrollo sostenible de una nación. Es importante acotar que, Ecuador al precisar su ranking en cuanto al manejo de la sostenibilidad empresarial ambiental, se observa en el siguiente cuadro una ubicación negativa.

\section{Cuadro $N^{\circ} 1$ Ranking sobre las Empresas y el Manejo Ambiental}

\begin{tabular}{lccc}
\hline País & Puesto & País & Puesto \\
\hline Estados Unidos & 1 & Chile & 28 \\
Alemania & 2 & Brasil & 58 \\
Finlandia & 3 & Colombia & 59 \\
Suiza & 4 & Perú & 71 \\
Dinamarca & 5 & Venezuela & 94 \\
Holanda & 6 & Tajikistan & 97 \\
Suecia & 7 & Nicaragua & 100 \\
Reino Unido & $\mathbf{8}$ & Ecuador & 105 \\
Japón & 9 & Bolivia & 117 \\
\hline Hong Kong & 10 & Paraguay & 120 \\
\hline
\end{tabular}

Fuente: Ruiz (ob.cit)

Las posiciones reflejadas en el cuadro que antecede, llevan a observar que Ecuador con respecto a los demás país, se encuentra ubicada en una posición desfavorable, para entender que las empresas que hacen vida en el territorio, se encuentran más interesadas en su capacidad económica, que manejar adecuadamente los diferentes aspectos que las hacen competitivas en el mundo. Además, la actuación de las mismas, se encuentra alejada de los criterios preestablecidos 


\section{Sostenibilidad empresarial en relación a los objetivos del desarrollo sostenible en el Ecuador}

Vol. 3, núm. 1., (2019)

Francisco Xavier Hugo Cárdenas; Carlos Roberto Flores Ramos; Álvaro Raúl Peralta Beltrán; Paul Eduardo Lara Pazos

en los objetivos de desarrollo sostenible propuesto por el gobierno, lo que invita a reflexionar y gestionar cambios específicos en cuanto a controles se refiere a fin de crear las condiciones ambientales para su crecimiento social.

En consecuencia, es importante destacar que la presencia de la sostenibilidad empresarial, obliga a los gerentes a registrar cambios en el paradigma gerencial, mirar la concepción del trabajo de una visión que proporcione satisfacción a los empleados, agilizar el trabajo en el marco de una actividad innovadora, competente, enmarcada en la excelencia como respuesta de cambio permanente, es decir, girar instrucciones que le permitan modificar los esquemas de aquella gestión tradicional, por una sostenible en pro de manifestar los cambios realmente enmarcados con las comunidades. Tal como se muestra en la siguiente figura.

\section{Figura $N^{\circ} 1$ Gestión Tradicional y Gestión Sostenible}

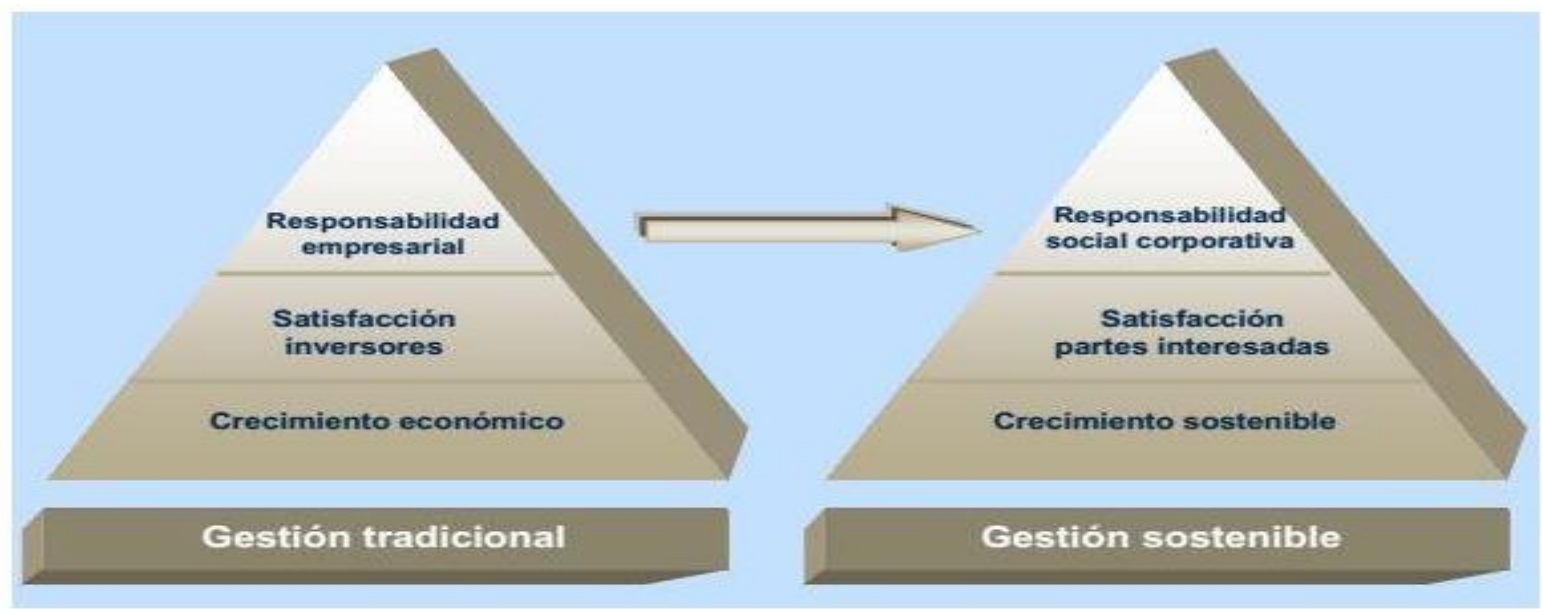

Fuente: Ruiz (ob.cit) 


\section{Sostenibilidad empresarial en relación a los objetivos del desarrollo sostenible \\ en el Ecuador}

Vol. 3, núm. 1., (2019)

Francisco Xavier Hugo Cárdenas; Carlos Roberto Flores Ramos; Álvaro Raúl Peralta Beltrán;

Paul Eduardo Lara Pazos

De acuerdo con la figura expuesta, se puede indicar que cuando las empresas se hacen sostenibles buscan no sólo llevar a cabo una gestión centrada en la satisfacción del recurso humano, sino mirar su responsabilidad social desde una visión corporativa, de este modo, hace correspondencia con los objetivos económicos, sociales, políticos y educativos trazados por el gobierno, pero en la medida que las posiciones gerenciales se encuentren determinadas por una visión tradicional, el trabajo se centra en lograr el progreso financiero y económico de la empresa, sin evaluar las consecuencias negativas que agrega a cada sector donde se encuentra insertada.

De las consideraciones indicadas anteriormente, se puede decir que la sostenibilidad empresarial, busca minimizar impactos, pues, es una forma de compromiso con el desarrollo sostenible, reconoce la importancia del desarrollo y crecimiento económico de la compañía de una manera socialmente responsable con sus comunidades y minimiza sus impactos en el medioambiente. El desarrollo sostenible considera el uso equitativo y responsable de los recursos, busca compatibilizar el desarrollo económico, social y ambiental de una comunidad a largo plazo, compatible con el desarrollo de la empresa. De este modo, la sostenibilidad empresarial permite el desarrollo de la organización e integrar herramientas, estrategias de gestión y de responsabilidad empresarial como elementos claves para asegurar la continuidad de su organización. Examina los impactos de la operación, funcionamiento y proyectos de crecimiento de la organización a sus grupos de interés, desde diversas perspectivas como son el medio ambiental, social y los aspectos financieros. 


\section{Sostenibilidad empresarial en relación a los objetivos del desarrollo sostenible en el Ecuador}

Vol. 3, núm. 1., (2019)

Francisco Xavier Hugo Cárdenas; Carlos Roberto Flores Ramos; Álvaro Raúl Peralta Beltrán; Paul Eduardo Lara Pazos

Por lo tanto, hoy más que nunca las diversas organizaciones y principalmente las empresas como agentes del crecimiento económico, además de orientar sus objetivos a la creación de valor, riqueza y empleo, deben integrar una operación socialmente responsable con las comunidades en las cuales se insertan. Esto debe fundamentarse en prácticas empresariales creíbles, éticas y transparentes para sustentar el negocio en mercados cada vez más exigentes, donde los grupos de interés demandan información de sostenibilidad de las empresas lo que además trae diversos beneficios para la propia organización. Para así, lograr insertar beneficios para la organización que están debidamente definidos con la visión y estrategias de desarrollo sostenible y de largo plazo, potenciamiento de sistemas de gestión, atracción y retención de clientes o usuarios y mantención y mejoramiento de la motivación, compromiso y productividad de trabajadores y proveedores.

Asimismo, se potencia la reputación de la empresa y la confianza de sus grupos de interés. A la vez que se atrae financiamiento, capital para su crecimiento y desarrollo, y se logran ventajas competitivas y posición de liderazgo. Actualmente muchas empresas implementan programas y proyectos de apoyo a la comunidad, no obstante es relevante que estas acciones respondan a los reales intereses y expectativas de los diversos grupos de interés de la empresa (clientes, trabajadores, comunidad, proveedores, entre otros), para generar una verdadera conexión con la estrategia de la empresa. La sostenibilidad se instaló inicialmente como acciones de responsabilidad social empresarial, que se orientaron a diversos tipos de acciones como transferencias a las comunidades, que no necesariamente responden a los aspectos estratégicos de la empresa. 


\section{Sostenibilidad empresarial en relación a los objetivos del desarrollo sostenible \\ en el Ecuador}

Vol. 3, núm. 1., (2019)

Francisco Xavier Hugo Cárdenas; Carlos Roberto Flores Ramos; Álvaro Raúl Peralta Beltrán;

Paul Eduardo Lara Pazos

Sin embargo, estas acciones se complementan con la creación del valor conjunto para la compañía y comunidad e integra acciones de innovación, las cuales varían en función a las habilidades empresariales y de gestión requieren mayor especialización. Lo que aprenden las propias empresas es que la sostenibilidad requiere reforzar valores como la ética, responsabilidad, respeto y transparencia en la organización de forma transversal. Ello permite desarrollar una operación y desempeño económico, social y ambiental consistente, y dar credibilidad al compromiso de sostenibilidad de la empresa genera mayores niveles de confianza en sus clientes, proveedores, trabajadores, inversionistas, entre otros grupos de interés.

\section{Objetivos del Desarrollo Sostenible en el Ecuador}

Con el fin de llevar a comprender la relación presente entre la sostenibilidad empresarial y el desarrollo sostenible en el Ecuador, se hace necesario hacer un recorrido por la perspectiva que condujo al gobierno en septiembre del 2015, conjuntamente con otros 193 países, adoptaron un compromiso histórico al aprobar los 17 Objetivos de Desarrollo Sostenible (ODS) que fueron consensuados después de más de 3 años de negociaciones y con la participación de todos los países miembros de la ONU, ONG y otros agentes.

Para el año 2000 se adoptaron 8 Objetivos de Desarrollo del Milenio (ODM) que fueron la promesa para defender los principios de dignidad humana, igualdad y equidad, y liberar al mundo de la pobreza extrema. El año 2015 era la fecha fijada para el cumplimiento de estos ODM, sin embargo no se lograron todas las metas establecidas. Por tal motivo, los Estados 


\section{Sostenibilidad empresarial en relación a los objetivos del desarrollo sostenible en el Ecuador}

Vol. 3, núm. 1., (2019)

Francisco Xavier Hugo Cárdenas; Carlos Roberto Flores Ramos; Álvaro Raúl Peralta Beltrán; Paul Eduardo Lara Pazos

renovaron su compromiso con el desarrollo social y la lucha contra la pobreza con la nueva agenda que traza una hoja de ruta para los próximos 15 años, bajo el nombre: Transformar nuestro mundo: la Agenda 2030 para el Desarrollo Sostenible.

Es importante destacar que, la apuesta de este compromiso será conseguir el desarrollo sostenible en el ámbito económico, social y ambiental de forma equilibrada e integrada, a través de 17 objetivos con 169 metas. Entre los nuevos Objetivos de Desarrollo Sostenible (ODS) se encuentran: erradicar la pobreza en todo el mundo; acabar con el hambre y lograr la seguridad alimentaria; garantizar una vida saludable para todos los seres humanos, así como una educación de calidad; conseguir la igualdad de género y el empoderamiento de mujeres y niñas; facilitar disponibilidad de agua en todo el mundo; reducir las desigualdades, entre otros.

- Objetivo 1 Fin de la Pobreza: Poner fin a la pobreza en todas sus formas en todo el mundo. Se espera reducir a la mitad la proporción de personas que viven en la pobreza y garantizar que todos los habitantes del planeta tengan los mismos derechos.

- Objetivo 2. Hambre Cero: Poner fin al hambre, lograr la seguridad alimentaria y la mejora de la nutrición y promover la agricultura sostenible. La nutrición deficiente provoca el $45 \%$ de las muertes de menores de 5 años.

- Objetivo 3. Salud y Bienestar: Garantizar una vida sana y promover el bienestar para todos en todas las edades. Una de las principales metas es reducir la tasa mundial de mortalidad materna a menos de 70 por cada 100.000 nacidos vivos. 


\section{Sostenibilidad empresarial en relación a los objetivos del desarrollo sostenible}

en el Ecuador

Vol. 3, núm. 1., (2019)

Francisco Xavier Hugo Cárdenas; Carlos Roberto Flores Ramos; Álvaro Raúl Peralta Beltrán;

Paul Eduardo Lara Pazos

- Objetivo 4 Educación de calidad: Garantizar una educación inclusiva, equitativa y de calidad y promover oportunidades de aprendizaje durante toda la vida para todos. Se velará por que todos los niños tengan una enseñanza primaria y secundaria completa, gratuita, equitativa y de calidad.

- Objetivo 5 Igualdad de Género: Lograr la igualdad entre los géneros y empoderar a todas las mujeres y las niñas; eliminando todas las formas de violencia contra todas las mujeres y las niñas en los ámbitos público y privado, incluidas la trata y la explotación sexual.

- Objetivo 6 Agua y Saneamiento: Garantizar la disponibilidad de agua y su gestión sostenible y el saneamiento para todas las personas en el mundo. 884 millones de personas en todo el mundo todavía no tienen acceso a agua potable.

- Objetivo 7 Energía Asequible y No Contaminante: Garantizar el acceso a una energía asequible, segura, sostenible y moderna para todos. Una de cada cinco personas no tienen acceso a la electricidad moderna.

- Objetivo 8. Trabajo decente y crecimiento económico: Promover el crecimiento económico sostenido, inclusivo y sostenible, el empleo pleno y productivo y el trabajo decente para todos. El fin es un crecimiento del producto interno bruto de al menos el 7\% anual en los países menos adelantados.

- Objetivo 9 Industria, Innovación e Infraestructura: Construir infraestructuras resilientes, promover la industrialización inclusiva y sostenible y fomentar la 


\section{Sostenibilidad empresarial en relación a los objetivos del desarrollo sostenible en el Ecuador}

Vol. 3, núm. 1., (2019)

Francisco Xavier Hugo Cárdenas; Carlos Roberto Flores Ramos; Álvaro Raúl Peralta Beltrán; Paul Eduardo Lara Pazos

innovación para apoyar el desarrollo económico y el bienestar humano, con especial hincapié en el acceso equitativo y asequible para todos.

- Objetivo 10 Reducción de las desigualdades: Reducir la desigualdad en y entre los países. Lograr progresivamente y mantener el crecimiento de los ingresos del $40 \%$ más pobre de la población a una tasa superior a la media nacional.

- Objetivo 11 Ciudades y Comunidades sostenibles: Lograr que las ciudades y los asentamientos humanos sean inclusivos, seguros, resilientes y sostenibles.

- Objetivo 12. Producción y consumo responsables: Garantizar modalidades de consumo y producción sostenibles para reducir a la mitad el desperdicio mundial de alimentos.

- Objetivo 13 Acción por el clima: Adoptar medidas urgentes contra el cambio climático mediante la educación y sensibilización de las personas y la negociación de acuerdos y medidas nacionales e internacionales para actuar todos juntos contra el cambio climático; minimizando su impacto en la vida de las personas.

- Objetivo 14.- Vida submarina: Conservar y utilizar en forma sostenible los océanos y mares. Desde hace 150 años, la acidificación de los océanos ha aumentado un 30\%, afectando a la vida marina. Por este motivo, para el año 2020 se espera al menos conservar por lo menos el $10 \%$ de las zonas costeras y marinas.

- Objetivo 15 Vida de ecosistemas terrestres: Proteger los bosques y luchar contra la desertificación, creando leyes para preservar los ecosistemas a nivel 


\section{Sostenibilidad empresarial en relación a los objetivos del desarrollo sostenible}

en el Ecuador

Vol. 3, núm. 1., (2019)

Francisco Xavier Hugo Cárdenas; Carlos Roberto Flores Ramos; Álvaro Raúl Peralta Beltrán;

Paul Eduardo Lara Pazos

mundial, reduciendo la degradación ambiental y conservando la diversidad biológica.

- Objetivo 16 Paz, justicia e instituciones sólidas: Promover sociedades pacíficas y mejor acceso a la justicia. Es necesario poner fin al maltrato, la explotación, la trata, la tortura y todas las formas de violencia, reduciendo las tasas de mortalidad.

- Objetivo 17 Alianzas para lograr los objetivos: Fortalecer y revitalizar la Alianza Mundial para el Desarrollo Sostenible. Solo Dinamarca, Luxemburgo, Noruega y Suecia y Reino Unido cumplen el compromiso de destinar un $0,7 \%$ de su ingreso nacional bruto a la ayuda oficial al desarrollo.

Cada uno de los objetivos destacado anteriormente, llevan a reflexionar que para lograrlos de manera efectiva, el Estado ecuatoriano, requiere una verdadera inserción de la sostenibilidad empresarial, en el campo social, educativo, político, cultural y económico en Ecuador, el gobierno busca mediante la realización de actividades mancomunadas con los países aliados, cambios significativos en materia de calidad de vida en la población ecuatoriana, para lo cual, busca disminuir la pobreza, reducir la desigualdad social, aumentar los niveles de vida, ofrecer espacios de salud que logren beneficiar a corto y largo plazo a toda la población.

Ante estas ideas, Briones (2018) destaca que en Ecuador la cobertura del:

Líquido por red pública creció del 66,4\% en 2014 al 71,9\% en 2017. Acceso a energía indicó que el país acomete un plan de eficiencia energética y mencionó que en el ODS 8, que versa sobre comunidades sostenibles, Ecuador redujo el déficit de 


\section{Sostenibilidad empresarial en relación a los objetivos del desarrollo sostenible en el Ecuador}

Vol. 3, núm. 1., (2019)

Francisco Xavier Hugo Cárdenas; Carlos Roberto Flores Ramos; Álvaro Raúl Peralta Beltrán; Paul Eduardo Lara Pazos

vivienda del 15,2 \% en 2014 al 13,4 \% en 2017. Se refirió también al objetivo 12 hay un superávit ecológico si se considera la biocapacidad del país y la huella ecológica; mientras que sobre el ODS 15, indicó que el país ha fortalecido la conservación de cuencas hidrográficas del 31,1 \% en 2014 al 32,6 \% en 2017. (p.2)

Estos datos, llevan a entender que el desarrollo sostenible en el Ecuador, muestra cambios significativos al garantizarle a la población beneficios colectivos e individuales, que les ayudan a tener mejores condiciones ambientales para el disfrute de sus derechos humanos universales, claro, está que dicho logra se encuentra estrechamente relacionado con la sostenibilidad que las empresas van afianzado en sus diferentes espacios geográficos y en función de ello, el Estado tiene oportunidad de ofrecer mejores oportunidades de vida a las comunidades.

En este orden de ideas, el desarrollo sostenible en el Ecuador, tiene sus acciones estimadas en una efectiva intervención por, parte de las empresas, quienes al considerar la sostenibilidad como parte esencial de sus objetivos, visión y misión, pueden conjuntamente trabajar en pro de darle respuesta oportuna a las necesidades de la población y con ello reducir los efectos que introducen los esquemas de una gestión empresarial tradicional.

\section{Conclusiones.}

Los aspectos desarrollados a lo largo del discurso teórico que caracteriza al presente artículo, permite indicar una serie de apreciaciones generales que sirven para valorar desde una perspectiva reflexiva la importancia del tema seleccionado; entre ellas se citan las siguientes: 


\section{Sostenibilidad empresarial en relación a los objetivos del desarrollo sostenible \\ en el Ecuador}

Vol. 3, núm. 1., (2019)

Francisco Xavier Hugo Cárdenas; Carlos Roberto Flores Ramos; Álvaro Raúl Peralta Beltrán;

Paul Eduardo Lara Pazos

Para que las empresas se desarrollen en el campo de la sostenibilidad es importante, que cada una conciba su visión organizacional desde tres vertientes: económico, ambiental y social, por ello, la conjugación de las tres hace posible establecer conexiones flexibles con las políticas estatales y así, garantizarle a la población en general una suma de beneficios básicos, especialmente aquellos grupos más vulnerables. En consecuencia, no pueden solo concebir sus funciones desde lo económico-financiero; sino, es significativo entender que el ambiente como espacio socializador debe ser cuidado y protegido, así como desarrollar planes o programas que ayuden a las comunidades en resolver sus problemas al tomar decisiones colectivas.

En consecuencia, cuando se incrementan las capacidades de sostenibilidad empresarial y en esa misma dirección el Estado de Ecuador, puede direccionar los objetivos propuestos con los otros países aliados, en pro de ofrecerle a la población una suma de beneficios colectivos que darán oportunidad a tener niveles óptimos de calidad de vida. Por ello, los gobiernos deben establecer una dinámica fiscal, externa e interna que le ayude a cada organización cumplir sus tareas en el marco de la recepción de los productos o servicios determinados por mantener interacciones básicas con las propuestas gubernamentales, de este modo, se consolida la relación con los objetivos de desarrollo sostenible en el Ecuador.

Cada uno de los objetivos de desarrollo sostenible en el Ecuador, están directamente vinculados con la visión, misión, objetivos y metas trazadas por las empresas, de allí, la significación que exista una correspondencia en su sostenibilidad para que la suma de acciones se conviertan en ejes esenciales que promocionan el hacer de cada objetivo y generar en las 


\section{Sostenibilidad empresarial en relación a los objetivos del desarrollo sostenible en el Ecuador}

Vol. 3, núm. 1., (2019)

Francisco Xavier Hugo Cárdenas; Carlos Roberto Flores Ramos; Álvaro Raúl Peralta Beltrán; Paul Eduardo Lara Pazos

comunidades beneficios generales en pro de garantizarle a la población sus derechos humanos, fijados por las sociedades.

No obstante, es importante que cada administrador de las empresas desarrolle las actividades de sostenibilidad, bajo una ética centrada en la solidaridad, tolerancia, cooperación como principios que norman la vida en sociedad y con ello, mejorar el entorno de las comunidades. Ante esto, las políticas de Estado deben concebir la gestión de las empresas desde la visión de sostenibilidad, propiciar cambios puntuales en la satisfacción de necesidades, reducir los esquemas de los paradigmas tradicionales, para así garantizar en las comunidades el alcance de los objetivos de desarrollo sostenible, y plantear en el tiempo cambios progresivos en la vida humana de los ecuatorianos y ecuatorianas.

Finalmente, se puede indicar que es importante elevar la conciencia empresarial en el Ecuador, solo mediante este accionar se podrá reconducir los cambios que buscan insertar los objetivos de desarrollo sostenible. Es decir, debe darse unas condiciones permeables que propicien encaminar los objetivos hacia su consecución y adecuar los espacios comunitarios a los nuevos esquemas de interés social. Esto conduce, a la necesidad de que los administradores de las empresas, conciban sus funciones bajo una perspectiva de integración económica-ambientalsocial.

\section{Bibliografía.}

Andrade, I. (2018). Beneficios de la Sostenibilidad Empresarial . Lutindex, 32-36.

Correa, R. (17 de Noviembre de 2015). https://www.cepalorga/.../cepal. Obtenido de https://www.cepalorga/.../cepal 


\section{Sostenibilidad empresarial en relación a los objetivos del desarrollo sostenible}

en el Ecuador

Vol. 3, núm. 1., (2019)

Francisco Xavier Hugo Cárdenas; Carlos Roberto Flores Ramos; Álvaro Raúl Peralta Beltrán; Paul Eduardo Lara Pazos

Ecuador, A. E. (24 de Abril de 2015). www.accionecologica.org/. Obtenido de www.accionecologica.org/

Ecuador, P. (8 de Enero de 2019). www.ec.undp.org/. Obtenido de www.ec.undp.org/

Gómez, C. (2018). Qué Indicadores Miden la Sostenibilidad Empresarial . Visión Gerencial , 5058.

J, B. (11 de Julio de 2018). Ecuador muestra la hoja de ruta de Cara a Objetivos de Desarrollo Sostenible. El Comercio, pág. https://www.elcomercio.com.

Mariño, P. (2018). Sostenibilidad Empresarial. IESA, 24-32.

Mendoza, F. (2017). Investigación Documental. España: Gedisa.

Naranjo, D. (2019). Método Científico. Caracas: Panapo.

Ruiz, V. (2017). Por que es Importante la Sostenibilidad. Administrativa, 22-25. 